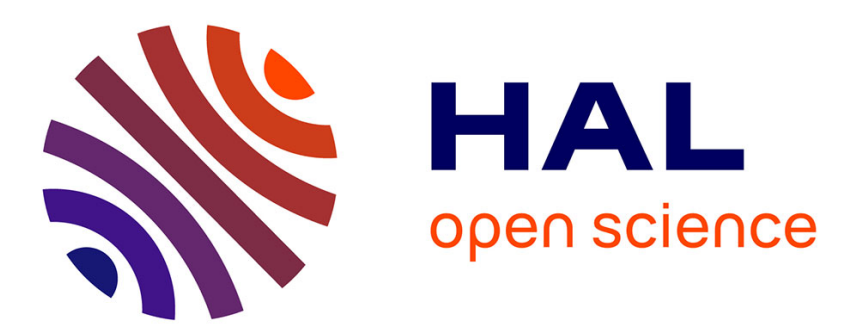

\title{
Uncertainty quantification for nonlinear reduced-order elasto-dynamics computational models
}

Evangéline Capiez-Lernout, Christian Soize, M Mbaye

\section{To cite this version:}

Evangéline Capiez-Lernout, Christian Soize, M Mbaye. Uncertainty quantification for nonlinear reduced-order elasto-dynamics computational models. IMAC-XXXIV, A Conference and Exposition on Structural Dynamics, SEM/IMAC, Jan 2016, Orlando, Fl, United States. pp.1-10. hal-01276798

\section{HAL Id: hal-01276798 \\ https://hal.science/hal-01276798}

Submitted on 20 Feb 2016

HAL is a multi-disciplinary open access archive for the deposit and dissemination of scientific research documents, whether they are published or not. The documents may come from teaching and research institutions in France or abroad, or from public or private research centers.
L'archive ouverte pluridisciplinaire $\mathbf{H A L}$, est destinée au dépôt et à la diffusion de documents scientifiques de niveau recherche, publiés ou non, émanant des établissements d'enseignement et de recherche français ou étrangers, des laboratoires publics ou privés. 


\title{
Uncertainty quantification for nonlinear reduced-order elasto-dynamics computa- tional models
}

\author{
E. Capiez-Lernout ${ }^{1}$, C. Soize ${ }^{1}$, M. Mbaye ${ }^{2}$ \\ ${ }^{1}$ Laboratoire Modélisation et Simulation Multi-Echelle, MSME UMR 8208 CNRS \\ Université Paris-Est, Marne-La-Vallée, France \\ ${ }^{2}$ Turbomeca, SAFRAN Group, Bordes, France
}

\begin{abstract}
The present work presents an improvement of a computational methodology for the uncertainty quantification of structures in presence of geometric nonlinearities. The implementation of random uncertainties is carried out through the nonparametric probabilistic framework from a nonlinear reduced-order model. With such usual modeling, it is difficult to analyze the influence of uncertainties on the nonlinear part of the operators with respect to its linear counterpart. In order to adress this problem, an approach is proposed to take into account uncertainties for both the linear and the nonlinear operators. The methodology is then validated in the context of the linear and nonlinear mistuning of an industrial integrated bladed-disk.
\end{abstract}

\section{KEYWORDS}

Mistuning — Geometric nonlinearities — Uncertainties - Reduced-order model - Structural dynamics

\section{Introduction}

Nowadays, an essential challenge consists in considering the geometric nonlinear effects in the computational dynamical models of structures. Such situations become realistic when the considered structures are slender or/and submitted to consequent loads. For exceptional operating regimes, the large strains and the large displacements cannot be neglected anymore (see for instance $[1,2,3]$ in the turbomachinery context or $[4,5]$ for thin or slender structures). Moreover, in many industrial applications, for which the investigated structures present a high complexity level, deterministic nonlinear computational models are in general not sufficient to accurately predict its dynamical behavior. Uncertainties have then to be taken into account in the computational models by using probabilistic models as soon as the probability theory can be used. Recently, a computational methodology adapted to the uncertainty quantification of structures in presence of geometric nonlinearities has been proposed and validated in the context of structural dynamics $[6,7,8]$. The implementation of random uncertainties is carried out through the nonparametric probabilistic framework from a nonlinear reduced-order model. It has been shown that such approach was adapted to the geometrical nonlinear case, by introducing a dedicated stiffness positive- definite operator self-containing the integrality of the information concerning the nonlinear geometric stiffness contributions. Nevertheless, its dimension increases with the square of the dimension of the nonlinear-reduced 
order model, requiring a large number of uncertain random germs. Consequently, establishing criteria for analyzing the influence of uncertainties on the nonlinear part of the operators with respect to its linear counterpart is difficult. The main idea for reducing the size of the random germ is to use a second reduction method before implementing the model of uncertainties. It is then possible to coherently take into account uncertainties on both the linear operator and the nonlinear operator. The paper is organized as follows. In Section 1, the main steps of the complete methodology is shortly summarized in order to give a global overview. This work is then focussed on the implementation of the random uncertainties. After recalling the usual way to implement uncertainties on the linear or nonlinear operators in the context of the nonparametric probabilistic method, it is pointed out that both situations do not appear to be compatible since no meaningful comparison can be made. A reasonable and logical requirement consists in using the same random germ for both situations, with a feasibility constraint controlled by the size of the random germ. This is achieved by replacing the Cholesky factorization of the dedicated stiffness positive-definite operator by another factorization obtained from a usual eigenvalue problem for which only the contributions related to the most contributing eigenvalues are kept. In Section 2, the methodology is applied on a large finite element model of a mistuned industrial centrifugal compressor.

\section{Computational modeling of mistuning uncertainties}

Recently, a complete methodology has been proposed for the geometric nonlinear analysis of mistuned bladed disks [8], for which all the terms including the rotational motion of the bladed-disk are taken into account. Below, the main steps of such methodology are briefly summarized.

1. Construction of the finite element model for the linear tuned case.

2. Solving the linear eigenvalue problem of the tuned structure for choosing the excitation frequency band known to yield drastic mistuning effects [9].

3. Construction of the external load in the time domain corresponding to a uniform sweep of the frequency band of excitation [8]

4. Choosing the projection basis as the linear eigenmodes of the tuned structure [10]

5. Direct construction of the mean nonlinear reduced-order model (MEAN-NL-ROM) by projection of the finite element operators on the chosen projection basis $[6,7]$

6. Implementing the mistuning through the nonparametric probabilistic framework [11] and controlling the mistuning level by dispersion parameter $\delta=\left(\delta_{M}, \delta_{D}, \delta_{C}, \delta_{K_{c}}, \delta_{K}\right)$, each scalar controlling the dispersion of the random mass, damping, gyroscopic coupling, centrifugal stiffness, elastic stiffness matrices.

7. Solving the stochastic nonlinear equations in the time-domain with efficient algorithms based on the arc-length methods $[7,8]$

All the details concerning the theoretical construction of such methodology and its application to a large finite element model of an industrial bladed-disk can be found in [8].

From now on, the work is more particularly focussed on point (6), which concerns the numerical modeling of stiffness mistuning uncertainties. 


\subsection{Linear mistuning with deterministic geometric nonlinearities}

Until now, it has been assumed in the industrial application [8] that the mistuning only affects the linear operators. In this case, that means that linear mistuning in presence of deterministic geometric nonlinearities is considered. Concerning the operators involved by the linear elastic constitutive equations, only the elastic linear stiffness operator issued from the MEAN-NL-ROM is uncertain, the quadratic and cubic terms remaining deterministic. Let $\left[\mathcal{K}^{(e)}\right]$ be the $(N \times N)$ elastic stiffness matrix issued from the MEAN-NL-ROM with positive-definite property. The corresponding random matrix $[\mathcal{K}]$ is then written as

$$
\left[\mathcal{K}^{(e)}\right]=\left[L_{K^{(e)}}^{\mathrm{T}}\right]\left[\mathbf{G}_{K^{(e)}}\left(\delta_{K^{(e)}}\right)\right]\left[L_{K^{(e)}}^{\mathrm{T}}\right],
$$

in which $\left[\mathcal{K}^{(e)}\right]$ is the $(N \times N)$ upper triangular matrix issued from the Cholesky factorization of $\left[\mathcal{K}^{(e)}\right]$, and where $\left[\mathbf{G}_{K^{(e)}}\left(\delta_{K^{(e)}}\right)\right]$ is a full random matrix with values in the set of all the positive-definite symmetric $(N \times N)$ matrices.

\subsection{Nonlinear mistuning}

From now on, we are interested in considering the case for which uncertainties affect all the operators issued from the elastic constitutive equations. This will be called nonlinear mistuning, that is to say both the linear and nonlinear stiffness operators are affected by uncertainties.

\subsubsection{Definition of a positive-definite stiffness operator}

In [12], the extension of the nonparametric probabilistic approach to the case of geometric nonlinearities has been considered. In such case, the $(P \times P)$ real matrix $[\mathcal{K}]$ with $P=N(N+1)$ is introduced as

$$
[\mathcal{K}]=\left[\begin{array}{cc}
{\left[\mathcal{K}^{(e)}\right]} & {\left[\widehat{\mathcal{K}}^{(2)}\right]} \\
{\left[\widehat{\mathcal{K}}^{(2)}\right]^{T}} & 2\left[\mathcal{K}^{(3)}\right]
\end{array}\right],
$$

in which the matrix blocks $\left[\widehat{\mathcal{K}}^{(2)}\right]$ and $\left[\mathcal{K}^{(3)}\right]$ are the $\left(N \times N^{2}\right)$ and the $\left(N^{2} \times N^{2}\right)$ real matrices resulting from the following reshaping operation

$$
\begin{gathered}
{\left[\widehat{\mathcal{K}}^{(2)}\right]_{\alpha J}=\widehat{\mathcal{K}}_{\alpha \beta \gamma}^{(2)}, J=(\beta-1) N+\gamma,} \\
{\left[\mathcal{K}^{(3)}\right]_{I J}=\mathcal{K}_{\alpha \beta \gamma \delta}^{(3)}, I=(\alpha-1) N+\beta, J=(\gamma-1) N+\delta .}
\end{gathered}
$$

It has been shown in [12] that matrix $[\mathcal{K}]$ is a symmetric and positive-definite matrix and can then be written as $[\mathcal{K}]=\left[L_{K}\right]^{T}\left[L_{K}\right]$. Consequently, the nonparametric probabilistic approach initially introduced in the linear context for positive-definite symmetric operators can easily be extended to the geometrically nonlinear context and the construction of random matrix $[\mathcal{K}]=\left[L_{K}\right]^{T}\left[\mathbf{G}_{K}\left(\delta_{K}^{N L}\right)\right]\left[L_{K}\right]$ is straightforward. Random matrix $[\mathcal{K}]$ can be block-decomposed similarly to Eq (2) so that the random linear elastic, quadratic and cubic random stiffness terms $\mathcal{K}_{\alpha \beta}^{(e)}, \widehat{\mathcal{K}}_{\alpha \beta \gamma}^{(2)}$ and $\mathcal{K}_{\alpha \beta \gamma \delta}^{(3)}$ can easily be deduced from Eqs.(2)-(4). Note that again, one scalar dispersion parameter controls the uncertainty level in the structure.

\subsubsection{Difficulties about quantifying nonlinear mistuning with respect to linear mistuning}

The main objective is to characterize the effects of the nonlinear mistuning with respect to the effects of the linear mistuning in presence of geometric nonlinearities. The nonlinear mistuning corresponds to the case for which all the linear and nonlinear operators are uncertain, in contrary to the linear mistuning where only the linear operators are uncertain. Both cases correspond to geometric nonlinear computations. 
(1) The case for which linear mistuning would be considered by generating random germ $\left[\mathbf{G}_{K^{(e)}}\left(\delta_{K^{(e)}}\right)\right]$ whereas the nonlinear mistuning would be treated by constructing $\left[\mathbf{G}_{K}\left(\delta_{K}^{N L}\right)\right]$ cannot be reasonably investigated. Indeed, the sizes of the random germs are thoroughly different and the input dispersion parameter would have to be characterized with respect to the other one.

(2) The case for which the same random germ $\left[\mathbf{G}_{K}\left(\delta_{K}^{N L}\right)\right]$ would have to be considered for both nonlinear and linear mistuning cases seems to be more coherent. This would imply that the linear mistuning would be considered by extracting the random linear part from random matrix $[\mathcal{K}]$, keeping the nonlinear quadratic and cubic terms as deterministic terms for the nonlinear calculations. Nevertheless, this case has been investigated in [7] in the context of the post-buckling nonlinear dynamical analysis of an uncertain cylindrical shell. With such method, a strong nonlinear level of uncertainty $\delta_{K}^{N L}=0.45$ yielded an equivalent linear uncertainty level $\delta_{K}^{L}=0.025$ for the linear part of the structure, yielding a subsequent scale effect, preventing to distinguish any differences between the linear and nonlinear uncertainty cases. Moreover, with such method, the nonlinear mistuning case would be involved with $N^{2}(N+1)(N+2) / 2 \ggg N(N+1) / 2$ independent random variables. This makes the feasibility of generating $\left[\mathbf{G}_{K}\left(\delta_{K}^{N L}\right)\right]$ questionable as $N$ increases.

Consequently, the following section proposes an improvement of the methodology, allowing both linear and nonlinear mistuning cases to be coherently considered.

\subsubsection{Strategy for modeling and quantifying the nonlinear mistuning}

In the present context, one wishes to obtain a coherent germ from matrix $[\mathcal{K}]$ for both linear and nonlinear mistuning cases. The idea is to perform a second reduced-order model of matrix $[\mathcal{K}]$ by solving first the single eigenvalue problem

$$
[\mathcal{K}] \boldsymbol{\psi}_{\alpha}=\lambda_{\alpha}^{\prime} \boldsymbol{\psi}_{\alpha}
$$

Matrix $[\mathcal{K}]$ can then be approximated by the $(P \times P)$ matrix $[\tilde{\mathcal{K}}]$ such that

$$
[\tilde{\mathcal{K}}]=\left[\tilde{L}_{K}\right]^{T}\left[\tilde{L}_{K}\right]
$$

in which $\left[\tilde{L}_{K}\right]$ is the $\left(N^{\prime} \times P\right)$ matrix defined by

$$
\left[\tilde{L}_{K}\right]=\left[\Lambda^{N^{\prime}}\right]^{\frac{1}{2}}\left[\Psi^{N^{\prime}}\right]^{T},
$$

where $\left[\Lambda^{N^{\prime}}\right]$ is the $\left(N^{\prime} \times N^{\prime}\right)$ diagonal matrix such that $\left[\Lambda^{N^{\prime}}\right]_{\alpha \alpha}=\lambda_{\alpha}^{\prime}$, where $\lambda_{1}^{\prime} \geq \lambda_{2}^{\prime} \geq \cdots \geq \lambda_{N^{\prime}}^{\prime}$ and where the columns of the $\left(P \times N^{\prime}\right)$ matrix $\left[\Psi^{N^{\prime}}\right]$ are the eigenvector $\boldsymbol{\psi}_{\alpha}, \alpha \in\left\{1, \ldots, N^{\prime}\right\}$ related to eigenvalues $\lambda_{\alpha}^{\prime}$. Random matrix $[\mathcal{K}]$ is then replaced by the random matrix $[\tilde{\mathcal{K}}]$ such that

$$
[\tilde{\mathcal{K}}]=\left[\tilde{L}_{K}\right]^{T}\left[\tilde{\mathbf{G}}_{K}\left(\delta_{K}^{N L}\right)\right]\left[\tilde{L}_{K}\right],
$$

in which $\left[\tilde{\mathbf{G}}_{K}\left(\delta_{K}^{N L}\right)\right]$ is a $\left(N^{\prime} \times N^{\prime}\right)$ random matrix with values in the set of the symmetric positive-definite matrices. It should be noted that one naturally expects to obtain $N \leq N^{\prime} \lll N(N+1)$ in order to propose a coherent quantification of the nonlinear mistuning with respect to the linear mistuning.

\subsection{Stochastic nonlinear reduced-order model}

Once all the operators of the MEAN-NL-ROM have been replaced by the properly random operators, including the strategy explained above concerning the nonlinear stiffness reduced operators, the stochastic nonlinear reduced-order model is given by the random field $\mathbf{U}^{N}(\cdot, t)$ that is written, for all $\mathbf{x} \in \boldsymbol{\Omega}$, as

$$
\mathbf{U}^{N}(\mathbf{x}, t)=\sum_{\beta=1}^{N} \varphi^{\beta}(\mathbf{x}) \mathbf{Q}_{\beta}(t)
$$


in which the $\mathbb{R}^{N}$-valued random variable $\mathbf{Q}(t)=\left(\mathbf{Q}_{1}(t), \cdots, \mathbf{Q}_{N}(t)\right)$ is solution of the following set of stochastic nonlinear differential equations,

$$
\begin{aligned}
& \mathcal{M}_{\alpha \beta} \ddot{\mathbf{Q}}_{\beta}+\left(\mathcal{D}_{\alpha \beta}+\mathcal{C}(\Omega)_{\alpha \beta}\right) \dot{\mathbf{Q}}_{\beta}+\left(\mathcal{K}_{\alpha \beta}^{(e)}+\mathcal{K}^{(c)}(\Omega)_{\alpha \beta}+\mathcal{K}_{\alpha \beta}^{(g)}\right) \mathbf{Q}_{\beta} \\
& +\mathcal{K}_{\alpha \beta \gamma}^{(2)} \mathbf{Q}_{\beta} \mathbf{Q}_{\gamma}+\mathcal{K}_{\alpha \beta \gamma \delta}^{(3)} \mathbf{Q}_{\beta} \mathbf{Q}_{\gamma} \mathbf{Q}_{\delta}=\mathcal{F}_{\alpha}
\end{aligned}
$$

The solution of such a stochastic nonlinear reduced-order model is calculated using the Monte Carlo numerical simulation, using an implicit and unconditionally stable integration scheme (Newmark method with the averaging acceleration scheme) combined with either the fixed point method or the Crisfield arc-length based algorithm $[13,8]$, depending on the nonlinearity rate.

\section{Results and Discussion}

\subsection{Description of the numerical model}

The structure under consideration is an industrial centrifugal compressor belonging to the class of integrated bladed disks. Due to proprietary reasons, the number $M$ of blades characterizing the order of the cyclic symmetry of the structure cannot be given. The finite element model of the structure is constructed with solid finite elements and is constituted of about 2,000,000 degrees of freedom. The structure is in rotation around its revolution axis with a constant velocity $\Omega=30,750 \mathrm{rpm}$. Since the dynamic analysis is carried out in the rotating frame of the structure, the rigid body motion due to the rotation of the structure corresponds to a fixed boundary condition at the inner radius of the structure. The bladed disk is made up of a homogeneous isotropic material. A modal damping model is added for the bladed disk.

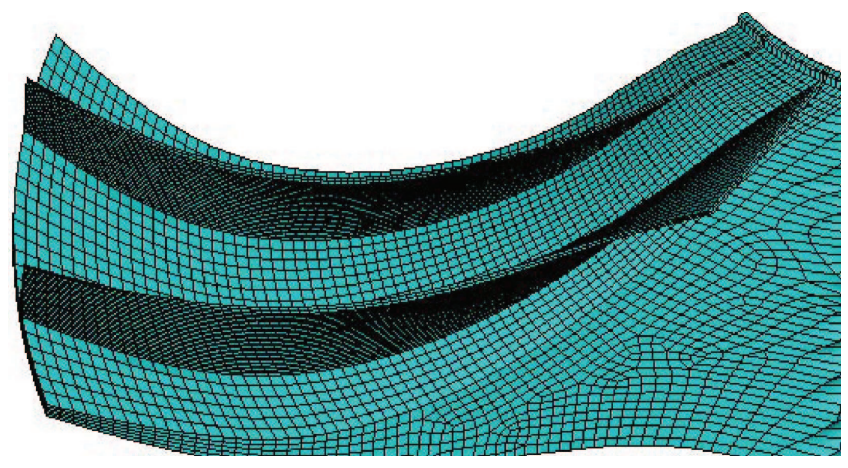

Fig. 1: Part of the finite element mesh of the bladed disk.

\subsection{Convergence of the stiffness operator issued from the MEAN-NL-ROM}

The numerical MEAN-NL-ROM related to the industrial bladed-disk is constructed using $N=60$ eigenmodes of vibration. Consequently, the matrix $[\mathcal{K}]$ defined by Eq. $(2)$ has dimension $(P \times P)$ with $P=3660$. The second reduction method is carried out as described in Section 1.2.3 and let $\left[\tilde{\mathcal{K}}^{N^{\prime}}\right]$ be the approximation of matrix $[\mathcal{K}]$ obtained with the $N^{\prime}$ most important eigenvalues. Figure $\mathbf{2}$ displays the graph $N^{\prime} \mapsto \operatorname{Conv}\left(N^{\prime}\right)$ in which

$$
\operatorname{Conv}(N)=\sqrt{\frac{\left\|\left[\tilde{\mathcal{K}}^{N}\right]-[\mathcal{K}]\right\|_{F}^{2}}{\|[\mathcal{K}]\|_{F}^{2}}}
$$

It can be shown that the convergence is fastly obtained. From now on, we choose $N^{\prime}=400$. 


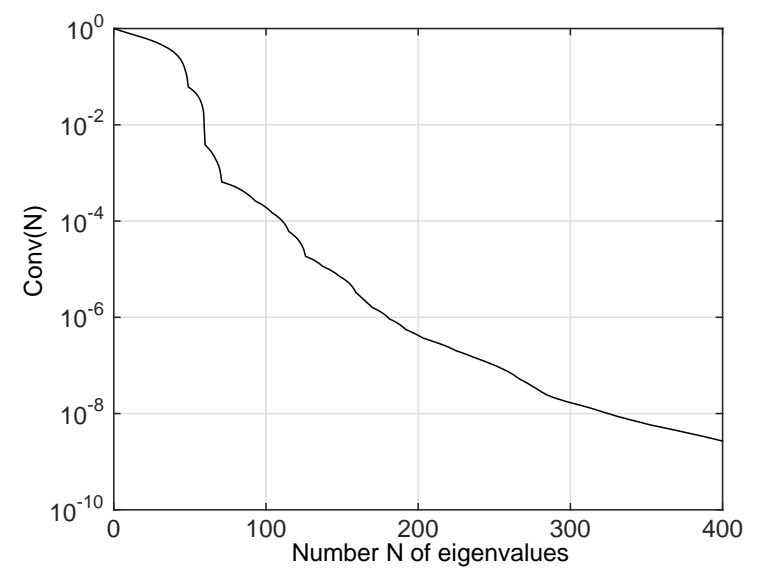

Fig. 2: Convergence analysis concerning the second reduction method: graph of function $N \mapsto \operatorname{Conv}(N)$.

\subsection{Convergence of the random response calculated with the second reduction method}

Let $\nu_{0}$ be the first eigenfrequency of the structure. From now on, all the quantities defined in the frequency domain are expressed with respect to $\nu / \nu_{0}$ in which $\nu$ is the physical frequency. The external load is a $5-t h$ engine-order excitation expressed in the time domain for which the corresponding dimensionless frequency excitation band is $\mathbb{B}_{\text {exc }}=[1.78,2.34]$ with an intensity load taken as $2.5 \mathrm{~N}$. It is located at the tip of each blade [14]. The random observation $W(2 \pi \nu)$ related to the STOCH-NL-ROM concerns the random displacement located at the tip of the most responding blade.

As shown in [8], it is seen that the geometric nonlinearities propagate the random response of the blade outside $\mathbb{B}_{\text {exc }}$. More particularly, it is seen that the resonance located outside $\mathbb{B}_{\text {exc }}$ has not only a nonnegligible contribution but also is particularly sensitive to uncertainties. Such complex dynamical behavior yields to introduce the two random observations $W_{\infty}^{1}$ and $W_{\infty}^{2}$ such that

$$
W_{\infty}^{1}=\max _{\mathbb{B}_{\mathrm{exc}}} W(2 \pi \nu) \quad W_{\infty}^{2}=\max _{\mathbb{B}_{\mathrm{sub}}} W(2 \pi \nu)
$$

in which $\mathbb{B}_{\text {sub }}=[1 ; 1.6]$ is a part of the frequency band of observation located below $\mathbb{B}_{\text {exc }}$. The convergence of the random solution is then analyzed with respect to the number $n_{s}$ of realizations required by the Monte Carlo numerical simulation and the size $N^{\prime}$ of the second-order reduction method by introducing the following quantity

$$
\operatorname{Conv}_{\infty}^{i}\left(N^{\prime}, n_{s}\right)=\sqrt{\frac{1}{n_{s}} \sum_{j=1}^{n_{s}}\left(\widetilde{W}_{\infty}^{N^{\prime}, i}\left(\theta_{j}\right)\right)^{2}}
$$

in which $\widetilde{W}_{\infty}^{N^{\prime}, i}\left(\theta_{j}\right)$ is the realization number $j$ of the approximation of random observation $W_{\infty}^{i}$ obtained with the second reduction method of size $N^{\prime}$. Figure 3 and $\mathbf{4}$ display the graphs $N^{\prime} \mapsto \operatorname{Conv}_{\infty}^{i}\left(N^{\prime}, 1000\right)$ and $n_{s} \mapsto \operatorname{Conv}_{\infty}^{i}\left(200, n_{s}\right)$. It can be concluded that a good approximation is obtained with $N^{\prime}=200$ and $n_{s}=600$. 


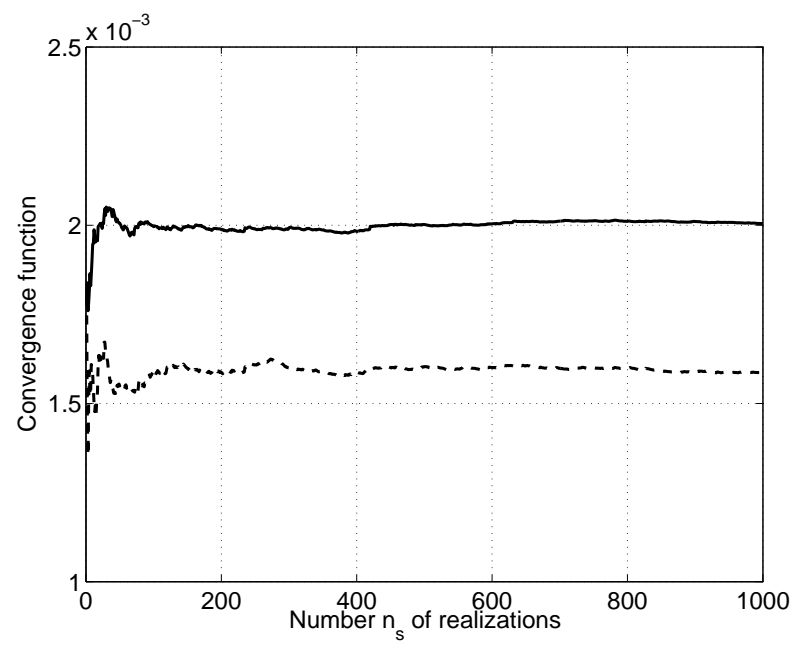

Fig. 3: Stochastic convergence analysis of the random response: graph $N^{\prime} \mapsto \operatorname{Conv}_{\infty}^{i}\left(N^{\prime}, 1000\right)$ (1) main resonance (solid line) (2) secondary resonance (dotted line).

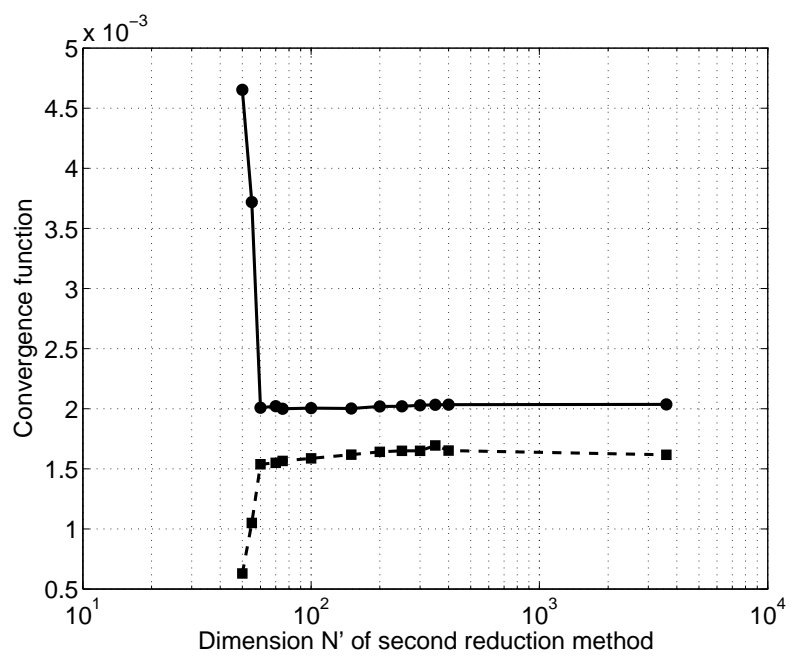

Fig. 4: Stochastic convergence analysis of the random response: $\operatorname{graph} n_{s} \mapsto \operatorname{Conv}_{\infty}^{i}\left(200, n_{s}\right)$ (1) main resonance (solid line) (2) secondary resonance (dotted line). 


\subsection{Quantification of the nonlinear mistuning with respect to the linear mistuning}

Figure 5 and $\mathbf{6}$ compare the random observation for the cases of nonlinear and linear mistuning respectively. There are slight differences between the two cases. It can be observed that the presence of uncertainties on the nonlinear part of the stiffness operator yield narrower confidence region. This means that the nonlinear random observation is more robust to uncertainties when uncertainties are taken into account on both the linear and nonlinear operators.

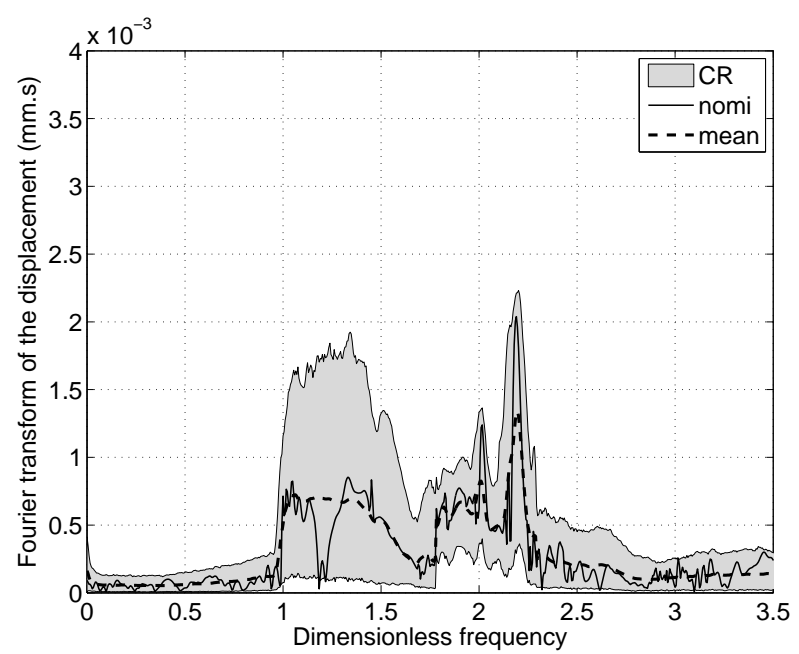

Fig. 5: Stochastic analysis for the nonlinear mistuning: random observation $W(2 \pi \nu)$ (1) mean model (thick line), mean of the stochastic model (thin dashed line), and confidence region(gray region).

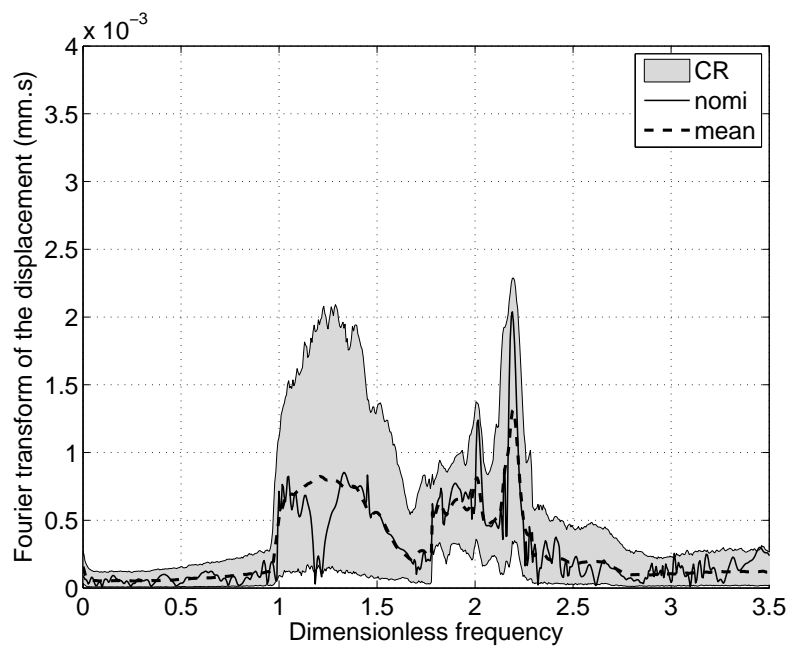

Fig. 6: Stochastic analysis for the linear mistuning: random observation $W(2 \pi \nu)(1)$ mean model (thick line), mean of the stochastic model (thin dashed line), and confidence region(gray region).

\section{Conclusions}

In the context of structural dynamics with geometric nonlinearities, a method has been presented for quantifying the propagation of uncertainties whether only the linear operators or both linear and nonlinear operators do have uncertainties. The methodology has been applied in the context of both the linear and 
nonlinear mistuning of an industrial integrated bladed-disk. It is shown that the presence of uncertainties on the nonlinear operators increase slightly the robustness of the random dynamical response.

\section{Acknowledgments}

This work was supported by the DGA (French defence procurement agency) in the context of the TURBODYNA project (project number ANR-13-ASTR-0008-01) related to the ANR ASTRID research program (specific support scheme for research works and innovation defence). SAFRAN Turbomeca is also acknowledged for giving permission to publish this work.

\section{References}

[1] P.F. Pai and A.H. Nayfeh. A Fully Nonlinear-Theory of Curved And Twisted Composite Rotor Blades Accounting For Warpings and 3-Dimensional Stress Effects. International Journal of Solids and Structures, 31(9):1309-1340, MAY 1994.

[2] D.-M. Tang and E.H. Dowell. Nonlinear response of a non-rotating rotor blade to a periodic gust. Journal of Fluids and Structures, 10(7):721-742, OCT 1996.

[3] A. Grolet and F. Thouverez. Free and forced vibration analysis of a nonlinear system with cyclic symmetry: Application to a simplified model. Journal of Sound and Vibration, 331(12):2911-2928, 2012.

[4] D.-H. Hodges, X.-Y. Shang, and C.E.S Cesnik. Finite element solution of nonlinear intrinsic equations for curved composite beams. Journal of the American Helicopter Society, 41(4):313-321, OCT 1996.

[5] H. Huang and Q. Han. Research on nonlinear postbuckling of functionally graded cylindrical shells under radial loads. Composite Structures, 92(6):1352-1357, MAY 2010.

[6] E. Capiez-Lernout, C. Soize, and M.-P. Mignolet. Computational stochastic statics of an uncertain curved structure with geometrical nonlinearity in three-dimensional elasticity. Computational Mechanics, 49(1):87-97, 2012.

[7] E. Capiez-Lernout, C. Soize, and Mignolet M.-P. Post-buckling nonlinear static and dynamical analyses of uncertain cylindrical shells and experimental validation. Computer Methods in Applied Mechanics and Engineering, 271:210-230, 2014.

[8] E. Capiez-Lernout, C. Soize, and M. Mbaye. Mistuning analysis and uncertainty quantification of an industrial bladed disk with geometrical nonlinearity. Journal of Sound and Vibration, 2015.

[9] S.-T. Wei and C. Pierre. Localization phenomena in mistuned assemblies with cyclic symmetry part ii: Forced vibrations. ASME Journal of vibration acoustics stress and reliability in design, 110(4):439-449, 1988.

[10] M.-P. Mignolet, A. Przekop, S.A. Rizzi, and M.S. Spottswood. review of indirect/non-intrusive reduced-order modeling of nonlinear geometric structures. Journal of Sound and Vibration, 332(10):2437-2460, 2013.

[11] C. Soize. Stochastic Models of Uncertainties in Computational Mechanics. Lecture Notes in Engineering Mechanics 2, American Society of Civil Engineers (ASCE), 2012. 
[12] M.-P. Mignolet and C. Soize. Stochastic reduced-order models for uncertain geometrically nonlinear dynamical systems. Computer Methods in Applied Mechanics and Engineering, 197:3951-3963, 2008 .

[13] M.A. Crisfield. Non-linear finite element analysis of solids and structures, Vol. 1 : essentials. John Wiley and Sons, Chichester, 1997.

[14] E. Capiez-Lernout, C. Soize, and M. Mbaye. Uncertainty quantification for an industrial mistuned bladed disk with geometrical nonlinearities. In paper GT2015-42471, Proceedings of the ASME Turbo Expo 2015: Turbine Technical Conference and Exposition GT2015, June 15-19, 2015, Montréal, Canada, 2015. 